\title{
Matching relations for decoupling in the standard model at two loops and beyond
}

\author{
Stephen P. Martin \\ Department of Physics, Northern Illinois University, DeKalb, Illinois 60115, USA
}

(Received 17 December 2018; published 14 February 2019)

\begin{abstract}
I discuss the matching relations for the running renormalizable parameters when the heavy particles (top quark, Higgs scalar, $Z$ and $W$ vector bosons) are simultaneously decoupled from the standard model. The complete two-loop-order matching for the electromagnetic coupling and all light fermion masses is obtained, augmenting existing results at four-loop order in pure QCD and in complete two-loop order for the strong coupling. I also review the further sequential decouplings of the lighter fermions (bottom quark, tau lepton, and charm quark) from the low-energy effective theory.
\end{abstract}

DOI: 10.1103/PhysRevD.99.033007

\section{INTRODUCTION}

The discovery of the Higgs scalar boson at the Large Hadron Collider has put the standard model of particle physics on a firm footing. At the same time, searches for physics beyond the standard model have not produced confirmed hints of any more fundamental structure. It therefore seems worthwhile to consider the standard model as quite possibly valid and complete up to well above the $\mathrm{TeV}$ energy scale, and to study its precise parameters and predictions, assuming that the next layer of fundamental new physics particles is heavy enough to be irrelevant at energy scales now within direct reach at colliders.

The standard model has within it an interesting hierarchy, with four fundamental particles (the top quark, the Higgs scalar, and the $Z$ and $W$ vector bosons) having masses within a factor of 2.2 of each other, and heavier than all others by well over an order of magnitude. This makes it sensible to consider a low-energy effective theory consisting of the $b, c, s, u, d$ quarks, the $\tau, \mu, e$ leptons, and their neutrinos, with renormalizable interactions coming from the unbroken $S U(3)_{c} \times U(1)_{\mathrm{EM}}$ gauge group, and nonrenormalizable four-fermion couplings to describe the weak interactions. This low-energy effective field theory can be matched onto the full $S U(3)_{c} \times S U(2)_{L} \times U(1)_{Y}$ high-energy theory with no particles decoupled, by considering common physical observables calculated in each theory in terms of parameters defined in the $\overline{\mathrm{MS}}$ renormalization scheme $[1,2]$ based on dimensional regularization [3-7].

Published by the American Physical Society under the terms of the Creative Commons Attribution 4.0 International license. Further distribution of this work must maintain attribution to the author(s) and the published article's title, journal citation, and DOI. Funded by SCOAP ${ }^{3}$.
In this paper, I will consider the decoupling relations that govern the matching at an arbitrary $\overline{\mathrm{MS}}$ renormalization scale, denoted by $Q$. Specifically, the pertinent running $\overline{\mathrm{MS}}$ parameters of the full standard model will be called

$$
\begin{aligned}
& g_{3}, \quad g, \quad g^{\prime}, \quad y_{t}, \quad y_{b}, \quad y_{c}, \quad y_{s}, \quad y_{u}, y_{d}, \\
& y_{\tau}, \quad y_{\mu}, \quad y_{e}, \lambda, \quad v \text {. }
\end{aligned}
$$

Here, $g_{3}, g$, and $g^{\prime}$ are the gauge couplings, the $y_{f}$ 's are the Yukawa couplings, $\lambda$ is the Higgs self-interaction coupling, and $v$ is the Higgs vacuum expectation value (VEV), defined in this paper as the minimum of the effective potential in the Landau gauge. This definition implies that scalar tadpole subgraphs vanish identically when summed to all orders in perturbation theory (including the tree-level tadpole), and so can be omitted from all Feynman diagrams. ${ }^{1}$ The very small effects of Cabibbo-KobayashiMaskawa mixing and neutrino masses are neglected. The running $\overline{\mathrm{MS}}$ squared masses of the standard model states are then denoted as follows:

$$
Z=\left(g^{2}+g^{2}\right) v^{2} / 4
$$

\footnotetext{
${ }^{1}$ The Landau gauge standard model effective potential and its minimization condition are presently known to full two-loop $[8,9]$ and three-loop [10,11] orders, and the four-loop part is known only at leading order in QCD [12]. These results make use of Goldstone boson resummation $[13,14]$ and employ three-loop vacuum integral basis functions defined and evaluated by Ref. [15]; for an alternative evaluation method, see Ref. [16]. In particular, Refs. [11,12] provide the formulas relating the VEV $v$ used here to the tree-level VEV $v_{\text {tree }}=\sqrt{-m_{H}^{2} / \lambda}$ used in many other works, which therefore must [17] include tadpole graphs. Outside of the Landau gauge, the effective potential is much more complicated at two-loop order [18], and is not known at threeloop order.
} 


$$
\begin{gathered}
W=g^{2} v^{2} / 4, \\
h=2 \lambda v^{2}, \\
t=y_{t}^{2} v^{2} / 2, \\
b=y_{b}^{2} v^{2} / 2, \quad \text { etc. }
\end{gathered}
$$

Due to the choice of the definition of the VEV $v$, these quantities are specific to the Landau gauge. As a matter of preference, I find the convenience (and increased accuracy) of not having tadpole graphs (with their associated $1 / \lambda$ factors in perturbation theory, coming from zero-momentum Higgs propagators) to be well worth the price of a Landaugauge-specific VEV and running masses, especially since these are not renormalization group scale-invariant observables anyway. The high-energy nondecoupled electromagnetic coupling is defined by

$$
e \equiv g g^{\prime} / \sqrt{g^{2}+g^{\prime 2}}
$$

In the low-energy $S U(3)_{c} \times U(1)_{\mathrm{EM}}$ effective field theory, the renormalizable $\overline{\mathrm{MS}}$ parameters will be denoted in this paper as

$$
\alpha_{S}, \alpha, m_{b}, m_{c}, m_{s}, m_{u}, m_{d}, m_{\tau}, m_{\mu}, m_{e} .
$$

To avoid confusion, $\alpha_{S}$ and $\alpha$ are only used for the low-energy effective theory, and never for the gauge couplings of the nondecoupled full standard model theory. Conversely, the symbols $g_{3}, g, g^{\prime}$, and $e$ are used exclusively to refer to quantities in the full nondecoupled theory. Note also that $\alpha$ is used in this paper to refer to the $\overline{\mathrm{MS}}$ quantity, not the so-called "on-shell" electromagnetic coupling. All of the parameters in Eqs. (1.1)-(1.8) depend on the $\overline{\mathrm{MS}}$ renormalization scale $Q$.

There are several complementary paths that one can take to relating these parameters to experimental results. In one approach, one makes direct use of low-energy experimental observables as the basic inputs, which then determine the parameters in Eq. (1.8), and then infers the full standard model parameters in Eq. (1.1) from them. In this paper, I will instead take the basic input parameters to be those of Eq. (1.1); then the low-energy observable data can be derived and used as the subjects of global fits. The purpose of this paper is limited to finding the matching relations that give the parameters of Eq. (1.8) as functions of those in Eq. (1.1). This will be done by treating the matching scale $Q$ as arbitrary, with the assumption that, typically, it should be chosen to be not much smaller than the $W$-boson mass and not much larger than the top quark mass, in order to avoid unnecessarily large logarithms. Note that $\ln \left(M_{t} / M_{W}\right)=$ 0.77 , so that any choice of $M_{W} \lesssim Q \lesssim M_{t}$ for the matching scale should be fine. (It is not necessary that each particle be automatically decoupled at the scale $Q$ equal to its mass, which is ambiguous in any case.)

Some observables, notably the pole masses of the top, Higgs, $Z$, and $W$, are only accessible in the high-energy theory. The Higgs boson mass has been connected to the self-coupling $\lambda$ including two-loop QCD corrections [19] and at full two-loop order in terms of interpolating formulas $[20,21]$. Analytical results and computer code for the Higgs mass at complete two-loop order have been presented in the tadpole-free scheme consistent with the present paper in Ref. [22], which also includes leading three-loop corrections, and in the scheme with a tree-level VEV and tadpoles in Refs. [23,24]. Multiloop corrections to the $W$ and $Z$ boson masses, their ratio (the $\rho$ parameter), and their relationships with other observables have been discussed in Refs. [2358]. In particular, Refs. [57,58] provide the complete twoloop analytic results for the $W$ and $Z$ pole masses, respectively, in the tadpole-free $\overline{\mathrm{MS}}$ scheme consistent with the conventions and notations of the present paper. For the top quark pole mass, the pure QCD contributions are known at the one-loop [59], two-loop [60], three-loop [61-63], and four-loop $[64,65]$ orders; these results also apply to the light quark pole masses in the decoupled theory. Contributions and uncertainty estimates from higher orders in QCD are discussed in Refs. [66-70]. The non-QCD one-loop corrections to fermion pole masses were given in Refs. [71,72]. Mixed two-loop QCD corrections to the top quark pole mass were obtained in Refs. [73-77], and the two-loop electroweak corrections in the "gaugeless" limit (where $W, Z$ masses are neglected compared to the top quark mass) are given in Refs. [78,79]. The full two-loop top quark pole mass corrections have been given in the tree-level VEV scheme in Ref. [23], and in the tadpole-free scheme used in the present paper in Ref. [80].

For computations at characteristic energies much lower or much higher than the matching scale, one should use the renormalization group equations to run the $\overline{\mathrm{MS}}$ parameters to an appropriate comparable $Q$, thus resumming the potentially large logarithms that would otherwise occur. For the full standard model, the beta functions are presently known at full two-loop [81-85] and three-loop [86-93] orders. The beta function for the Higgs self-coupling is also known at four loops in the leading order in QCD [12,94]. For the strong gauge coupling, the pure QCD contributions to the beta function are known at the four-loop $[95,96]$ and five-loop [97,98] orders, and the QCD contributions to the beta functions of the quark Yukawa couplings (or equivalently, the running quark masses) are likewise known at the three-loop [99], four-loop [100,101], and five-loop [102] orders. These QCD results also apply to the $\alpha_{S}$ and quark masses of the low-energy effective theory, by changing the variable number of active quarks.

There are also already extensive multiloop results on the decoupling matching relations involving the strong interactions. The one-loop and two-loop decouplings of the 
QCD coupling at quark thresholds were discussed long ago in Refs. [103-105], respectively. The pure QCD three-loop and four-loop threshold corrections for $\alpha_{S}$ were obtained in Refs. [106-109], respectively. The complete two-loop threshold corrections for $\alpha_{S}$ including electroweak and top quark Yukawa effects were given in Ref. [110] and have been checked as part of the present work. For the pure QCD contributions to quark mass threshold relations, the threeloop results were obtained in Refs. [106,107], and the fourloop results in Ref. [111]. All of the pure QCD results for the running and decoupling of $\alpha_{S}$ and quark masses have been incorporated into the RunDec [112] computer software packages.

The electromagnetic coupling is usually related to the very precisely known low-energy Thomson scattering value $\alpha_{\text {Thomson }}=1 / 137.0359991 \ldots$ as the basic input parameter, through radiative corrections to the photon self-energy function $[23,25,32,56,113-126]$. The bottleneck to accuracy in running $\alpha$ to very high energies (where it can be matched to $g, g^{\prime}$ ) comes from the nonperturbative hadronic contributions, often parametrized as $\Delta \alpha_{\text {had }}^{(5)}\left(m_{Z}\right)$. For recent evaluations of this important quantity, see Refs. [127-129] and references therein. In this paper, I will instead concentrate on the connection to the far-ultraviolet, fundamental definition of the standard model by obtaining the complete two-loop relationship between the $\overline{\mathrm{MS}}$ parameters $g, g^{\prime}, \ldots$ of the standard model and the $\overline{\mathrm{MS}}$ running coupling $\alpha(Q)$ in the low-energy theory when $t, h, Z, W$ are simultaneously decoupled. $^{2}$ The relationship between $\alpha(Q)$ and the very low-energy input $\alpha_{\text {Thomson }}$ is in this paper left as a separate issue, as addressed in Refs. [23,25,32,56,113-126].

The other new result to be obtained below is the complete two-loop matching for all of the light fermion masses listed in Eq. (1.8). The relation between the Yukawa couplings and the pole masses of the lightest five quarks were obtained to order $\alpha_{S} \alpha$ in Ref. [130]. In Ref. [79], the relationship between the bottom quark on-shell mass and its Yukawa coupling and running mass were obtained at two-loop order in the gaugeless limit, for both a tree-level VEV scheme and an "on-shell" definition of the VEV, $v_{\text {on-shell }}^{2} \equiv 1 / \sqrt{2} G_{F}$. This has been extended to full twoloop order in Ref. [23], with results given in terms of numerical linear interpolation formulas. In Ref. [131], the matching formulas for decoupling were given for the

\footnotetext{
${ }^{2}$ Note, however, that the $\hat{\alpha}^{(5)}\left(M_{Z}\right)$ quoted as the $\overline{\mathrm{MS}}$ coupling in the Review of Particle Properties (RPP) [126] decouples the top quark but not the $W$ boson, and so is not the same as the $\overline{\mathrm{MS}}$ scheme $\alpha(Q)$ as defined here within the five-quark, three-lepton $S U(3)_{c} \times U(1)_{\mathrm{EM}}$ gauge theory. In fact, strictly speaking, $\hat{\alpha}^{(5)}$ as defined in the RPP (following Refs. [113,115]) is not really an $\overline{\mathrm{MS}}$ coupling in the usual sense, because once the top quark has been decoupled, $S U(2)_{L}$ gauge invariance is explicitly and irretrievably broken, so that the $W, Z$ bosons should also be decoupled in order to have a renormalizable effective theory.
}

bottom quark mass, again using numerical interpolation formulas. In this paper, I will give the analytic results for the matching relations for the bottom quark as well as all other light quark masses, using the tadpole-free scheme to define the standard model VEV (and thus the running masses) in the nondecoupled theory.

The method used to find each decoupling matching relation is to compute a gauge-invariant physical quantity two ways, in terms of the parameters of the decoupled and the nondecoupled theories, and then require that the results agree. For the gauge couplings, the physical quantity is the residue of the pole in a scattering amplitude at $p^{2}=0$, where $p^{\mu}$ is the four-momentum of the gauge boson mediating the interaction. In the case of the fermion masses $m_{f}$, the physical quantity is the pole mass. The methods used here for the necessary calculations are very similar to those in Refs. [11,22,57,58,80], and all notations are chosen to be consistent with those papers. In particular, logarithms involving the renormalization scale will be denoted by

$$
\overline{\ln }(x) \equiv \ln \left(x / Q^{2}\right)
$$

where $x=t, h, Z, W, \ldots$ are $\overline{\mathrm{MS}}$ squared masses. In the following, only vacuum graphs occur in the final results, so they can be written in terms of $\overline{\ln }(x)$ and the two-loop renormalized vacuum basis integral function $[8,132]$. The notation used here is, in terms of dilogarithms, for $x, y \leq z$,

$$
\begin{aligned}
I(x, y, z)= & \frac{1}{2}(x-y-z) \overline{\ln }(y) \overline{\ln }(z)+\frac{1}{2}(y-x-z) \overline{\ln }(x) \overline{\ln }(z) \\
& +\frac{1}{2}(z-x-y) \overline{\ln }(x) \overline{\ln }(y) \\
& +2 x \overline{\ln }(x)+2 y \overline{\ln }(y)+2 z \overline{\ln }(z)-\frac{5}{2}(x+y+z) \\
& +r\left[\operatorname{Li}_{2}\left(k_{1}\right)+\operatorname{Li}_{2}\left(k_{2}\right)-\ln \left(k_{1}\right) \ln \left(k_{2}\right)\right. \\
& \left.+\frac{1}{2} \ln (x / z) \ln (y / z)-\zeta_{2}\right],
\end{aligned}
$$

with $r=\sqrt{\lambda(x, y, z)}$ and $k_{1}=(z+x-y-r) / 2 z$ and $k_{2}=(z+y-x-r) / 2 z$, where

$$
\lambda(x, y, z) \equiv x^{2}+y^{2}+z^{2}-2 x y-2 x z-2 y z .
$$

The function $I(x, y, z)$ implicitly depends on $Q$ through the $\overline{\ln }$ functions, and it is symmetric under the interchange of any of its arguments $x, y, z$. Some useful special cases are as follows:

$$
I(0,0, x)=-\frac{1}{2} x \overline{\ln }^{2}(x)+2 x \overline{\ln }(x)-\frac{5}{2} x-\zeta_{2} x,
$$




$$
\begin{gathered}
I(0, x, x)=-x \overline{\ln }^{2}(x)+4 x \overline{\ln }(x)-5 x, \\
I(0, x, y)=(y-x)\left[\operatorname{Li}_{2}(1-y / x)+\frac{1}{2} \overline{\ln }^{2}(x)\right] \\
+y \overline{\ln }(y)[2-\overline{\ln }(x)]+2 x \overline{\ln }(x)-\frac{5}{2}(x+y) .
\end{gathered}
$$

For use below in the matching relations for gauge couplings, it is convenient to define a $Q$-independent combination function:

$$
\begin{aligned}
F(x, y) \equiv & I(x, x, y)+(x-y / 2) \overline{\ln }^{2}(x)+y \overline{\ln }(x) \overline{\ln }(y) \\
& +(4 x-2 y) \overline{\ln }(x)-8 x \overline{\ln }(y) \\
& +\left[(4 x-y)^{2} / 6 x\right] \ln (y / x) \\
& -x / 3+31 y / 6-y^{2} / 3 x
\end{aligned}
$$

which has the nice property that the following limit is finite:

$$
\lim _{y \rightarrow 4 x} F(x, y) /(y-4 x)^{3}=[2 \ln (2)-1] / 60 x^{2} .
$$

Although the definitions in terms of ordinary dilogarithms are convenient for computer numerical evaluation, it is perhaps worth noting that for $y \leq 4 x$, one can also write

$$
\begin{aligned}
F(x, y)= & (4 x-y)\left[\frac{1}{2} \Phi(y / 4 x)+\left(\frac{4}{3}+\frac{y}{6 x}\right) \ln (x / y)\right. \\
& \left.-\frac{4}{3}+\frac{y}{3 x}\right]
\end{aligned}
$$

where

$$
\Phi(z)=4 \sqrt{\frac{z}{1-z}} \mathrm{Cl}_{2}(2 \arcsin (\sqrt{z})),
$$

with the Clausen integral function defined by

$$
\mathrm{Cl}_{2}(x)=-\int_{0}^{x} d t \ln (2 \sin (t / 2))
$$

\section{DECOUPLING RELATIONS IN THE STANDARD MODEL}

Consider the simultaneous decoupling of $t, h, Z$, and $W$ from the standard model at a scale $Q$. The matching relations for the low-energy effective theory renormalizable parameters in the $\overline{\mathrm{MS}}$ scheme can be written as

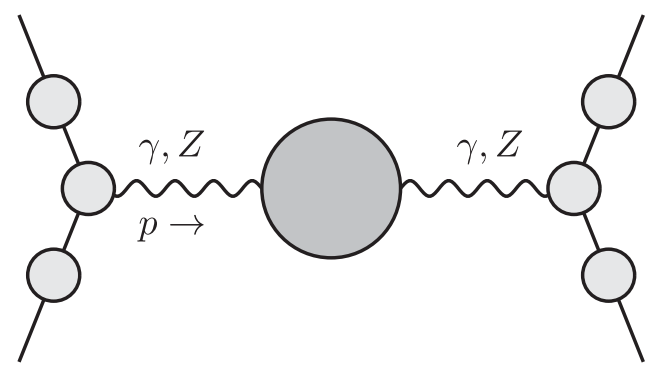

FIG. 1. The decoupling matching relation for $\alpha$ in the standard model is obtained from the residue of the pole at $p^{2}=0$ in the amplitude for neutral current scattering of charged particles, represented by the straight lines. By choosing the scattering charged particles to be vectorlike singlets under $S U(2)_{L}$ and to have infinitesimal $U(1)_{Y}$ charges, the one-particle irreducible vertex corrections and external state propagator corrections (depicted as the smaller light gray blobs) are parametrically suppressed by an arbitrary amount, so that only the mixed $\gamma, Z$ propagator corrections (larger, darker gray blob) contribute.

$$
\begin{gathered}
\alpha=\frac{e^{2}}{4 \pi}\left[1+\sum_{\ell=1}^{\infty} \frac{1}{\left(16 \pi^{2}\right)^{\ell}} \theta_{\alpha}^{(\ell)}\right], \\
\alpha_{S}=\frac{g_{3}^{2}}{4 \pi}\left[1+\sum_{\ell=1}^{\infty} \frac{1}{\left(16 \pi^{2}\right)^{\ell}} \theta_{\alpha_{S}}^{(\ell)}\right], \\
m_{f}=\frac{y_{f} v}{\sqrt{2}}\left[1+\sum_{\ell=1}^{\infty} \frac{1}{\left(16 \pi^{2}\right)^{\ell}} \theta_{m_{f}}^{(\ell)}\right], \\
(f=b, c, s, u, d, \tau, \mu, e),
\end{gathered}
$$

where the $\ell$-loop contributions $\theta_{X}^{(\ell)}$ on the right sides are functions of the parameters $g_{3}, g, g^{\prime}, y_{t}, v$, and the matching renormalization scale $Q$. The effects of $y_{f}$ for $f \neq t$ are negligible and therefore neglected, except of course for the leading factor of $y_{f}$ in Eq. (2.3). Results below are expressed $^{3}$ in terms of the running $\overline{\mathrm{MS}}$ squared masses defined in Eqs. (1.2)-(1.5).

\section{A. Matching of $\alpha$}

To determine the matching condition for $\alpha$ in the lowenergy theory, consider the residue of the pole at $p^{2}=0$ in the neutral current channel amplitude for the scattering of charged particles, as depicted in Fig. 1. This is done first in the full standard model including both $\gamma$ and $Z$ contributions to the neutral current, and then in the low-energy

\footnotetext{
${ }^{3}$ It is also easy to reexpress these results in terms of pole squared masses $M_{h}^{2}, M_{W}^{2}, M_{Z}^{2}, M_{t}^{2}$, by using the one-loop expressions relating them to $h, W, Z, t$ (found, e.g., in Refs. $[22,57,58,80]$, in the notations and VEV convention of the present paper), but that will not be done explicitly here.
} 
effective theory where only $\gamma$ exchange contributes. Requiring that the results of the two calculations agree gives the matching condition.

In order to avoid complications involving charged particle propagator and vertex corrections, it is convenient to use a trick by taking the charged particles to be vectorlike singlets under weak isospin $S U(2)_{L}$ and to carry infinitesimal electric charges, which are therefore also equal to their $U(1)_{Y}$ charges. This ensures that the one-particleirreducible vertex corrections and the charged particle propagator corrections to the amplitude are parametrically suppressed by an arbitrary amount relative to the bosonic propagator corrections, due to higher powers of the infinitesimal charges, and so they can be neglected. The result for the matching of the electromagnetic coupling then follows only from consideration of the corrections to the $\gamma, Z$ system propagator. The idea behind this trick is that $U(1)_{\mathrm{EM}}$ gauge invariance guarantees that the matching condition for the electromagnetic coupling cannot depend on the quantum numbers of the charged states to which the neutral current couples, so the same result must obtain for other scattering processes involving chiral fermions including $S U(2)_{L}$ doublets such as those in the standard model, where vertex and fermion propagator corrections are nontrivial.

The propagator matrix for the $\gamma, Z$ system can be written in terms of the components of the (transverse) oneparticle-irreducible self-energy functions $\Pi_{a b}(s)$ for $a, b=$ $\gamma, Z$ and $s=-p^{2}$, as $i G\left(\eta^{\mu \nu}-p^{\mu} p^{\nu} / p^{2}\right)$, where

$$
G^{-1}=\left(\begin{array}{cc}
s-\Pi_{\gamma \gamma}(s) & -\Pi_{\gamma Z}(s) \\
-\Pi_{\gamma Z}(s) & s-m_{Z}^{2}-\Pi_{Z Z}(s)
\end{array}\right) .
$$

It follows that

$$
\begin{gathered}
G_{\gamma \gamma}=\frac{1}{s-\tilde{\Pi}_{\gamma \gamma}}, \\
G_{\gamma Z}=\frac{\Pi_{\gamma Z}}{\left(s-m_{Z}^{2}-\Pi_{Z Z}\right)\left(s-\tilde{\Pi}_{\gamma \gamma}\right)}, \\
G_{Z Z}=\frac{s-\Pi_{\gamma \gamma}}{\left(s-m_{Z}^{2}-\Pi_{Z Z}\right)\left(s-\tilde{\Pi}_{\gamma \gamma}\right)},
\end{gathered}
$$

where

$$
\tilde{\Pi}_{\gamma \gamma} \equiv \Pi_{\gamma \gamma}+\left(\Pi_{\gamma Z}\right)^{2} /\left(s-m_{Z}^{2}-\Pi_{Z Z}\right)
$$

Now, the neutral current interaction amplitude between two $S U(2)_{L}$ singlet states with infinitesimal $U(1)_{Y}$ charges is just proportional to
$\mathcal{A}=g^{\prime 2} G_{Y Y}=g^{\prime 2}\left[c_{W}^{2} G_{\gamma \gamma}-2 c_{W} s_{W} G_{\gamma Z}+s_{W}^{2} G_{Z Z}\right]$,

where $c_{W}=g / \sqrt{g^{2}+g^{\prime 2}}$ and $s_{W}=g^{\prime} / \sqrt{g^{2}+g^{\prime 2}}$. It follows that

$\mathcal{A}=e^{2}\left[1+\frac{\left(g^{\prime} / g\right)^{2}\left(s-\Pi_{\gamma \gamma}\right)-\left(2 g^{\prime} / g\right) \Pi_{\gamma Z}}{s-m_{Z}^{2}-\Pi_{Z Z}}\right] /\left[s-\tilde{\Pi}_{\gamma \gamma}\right]$.

The existence of a massless photon pole in the amplitude at $s=0$ therefore implies

$$
\tilde{\Pi}_{\gamma \gamma}(0)=0,
$$

and the residue of the pole in $\mathcal{A}$ is a gauge-invariant physical observable:

$\operatorname{res}(\mathcal{A})=e^{2}\left[1+\frac{\left(g^{\prime} / g\right) \Pi_{\gamma Z}(0)}{m_{Z}^{2}+\Pi_{Z Z}(0)}\right]^{2} /\left[1-\tilde{\Pi}_{\gamma \gamma}^{\prime}(0)\right]$.

Here Eq. (2.11) has been used to eliminate $\Pi_{\gamma \gamma}(0)$ from the numerator. So far, no perturbative expansions or approximations or assumptions about the particular choice of gauge-fixing scheme have been used.

The calculation of the $\Pi_{a b}(s)$ is then performed in the Landau gauge in a loop expansion in terms of bare parameters (with no counterterm diagrams) in $d=4-$ $2 \epsilon$ dimensions, and at the end, the result for the residue of the pole, $\operatorname{res}(\mathcal{A})$, is translated to the $\overline{\mathrm{MS}}$ scheme by the standard parameter redefinitions that give bare parameters (including the VEV) in terms of $\overline{\mathrm{MS}}$ ones. ${ }^{4}$ This procedure is simpler and easier than using separate counterterm Feynman rules from the start, and the cancellation of poles in $\epsilon$ provides a check. The verification of Eq. (2.11) through two-loop order gives another check.

The calculation of $\operatorname{res}(\mathcal{A})$ is then repeated in the low-energy theory with $t, h, Z, W$ absent, and therefore no $\Pi_{\gamma Z}$ or $\Pi_{Z Z}$, so that $\Pi_{\gamma \gamma}(0)=0$, and $\operatorname{res}(\mathcal{A})=4 \pi \alpha /$ $\left[1-\Pi_{\gamma \gamma}^{\prime}(0)\right]$. Requiring that the two results for the observable $\operatorname{res}(\mathcal{A})$ be equal gives the matching condition for the electromagnetic coupling, after taking into account the oneloop matching for the light fermion masses between the two theories, from Sec. II C below. Note that nonperturbative effects from confined light quarks are common to the two versions of $\operatorname{res}(\mathcal{A})$, and so cancel out.

At one-loop order, one obtains the well-known result

$$
\theta_{\alpha}^{(1)}=e^{2}\left[\frac{2}{3}-7 \overline{\ln }(W)+\frac{16}{9} \overline{\ln }(t)\right] .
$$

\footnotetext{
${ }^{4}$ In the same notations and conventions as the present paper, they can be found in Eqs. (2.5)-(2.24) of Ref. [22], Eqs. (2.3)(2.12) of Ref. [57], and Eqs. (2.5)-(2.8) of Ref. [58].
} 
For the two-loop contribution to the matching, I obtain

$$
\begin{aligned}
\theta_{\alpha}^{(2)}= & e^{2} g_{3}^{2}\left[-\frac{64}{9} \overline{\ln }(t)-\frac{208}{27}\right]+e^{2} y_{t}^{2}\left\{\frac{16 t(h-t)}{3 h(4 t-h)^{2}} F(t, h)-\frac{16 t}{9 h}[1+\ln (h / t)]\right. \\
& +\frac{16 t}{9(4 t-Z)^{3}}\left[t\left(80 W / Z-7-64 W^{2} / Z^{2}\right)+8 Z-40 W+32 W^{2} / Z\right] F(t, Z) \\
& \left.+4\left[\left(80 W / Z-64 W^{2} / Z^{2}\right)[1+\ln (Z / t)]+2+3 \overline{\ln }(h)-7 \overline{\ln }(Z)-14 \overline{\ln }(t)\right] / 27+22 \overline{\ln }(t)-\frac{43}{4}\right\} \\
& +e^{2} g^{2}\left\{\frac{3 W\left(3 h^{2}-12 h W+4 W^{2}\right)}{h(4 W-h)^{3}} F(W, h)+\left(\frac{W}{h}-2\right) \overline{\ln }(h)+\frac{9 W\left(4 W^{2}-4 W Z+3 Z^{2}\right)}{Z^{2}(4 W-Z)^{2}} F(W, Z)\right. \\
& +\left(\frac{661 Z}{108 W}-\frac{491}{27}+\frac{319 W}{27 Z}+\frac{12 W^{2}}{Z^{2}}\right) \overline{\ln }(Z)+\left(\frac{20}{3}+\frac{37 W}{3 Z}-\frac{12 W^{2}}{Z^{2}}-\frac{W}{h}\right) \overline{\ln }(W)+\frac{5 t}{3(t-W)} \ln (t / W) \\
& \left.+\frac{31}{81}-\frac{3 h}{4 W}+\frac{W}{h}+\frac{12 W^{2}}{Z^{2}}-\frac{799 W}{27 Z}-\frac{1057 Z}{324 W}\right\}+e^{4}\left\{49 \overline{\ln }^{2}(W)-\frac{224}{9} \overline{\ln }(t) \overline{\ln }(W)+\frac{256}{81} \overline{\ln }^{2}(t)\right\} .
\end{aligned}
$$

The $g_{3}^{2}$ part of Eq. (2.14) can be checked to be consistent with previously known results for the relation between the Thomson scattering value of $\alpha$ and its $\overline{\mathrm{MS}}$ version, e.g., in Ref. [23].

The presentation of Eq. (2.14) is made simpler by the use of the function $F(x, y)$ defined in Eq. (1.15) above. Equation (1.16) shows immediately that $\theta_{\alpha}^{(2)}$ is finite and well defined for $h=4 t$ and $Z=4 t$ and for $h=4 W$ and $Z=4 W$, as well as for $t=W$, despite the presence of denominators proportional to $(4 t-h)^{2}$ and $(4 t-Z)^{3}$ and $(4 W-h)^{3}$ and $(4 W-Z)^{2}$ and $t-W$ in Eq. (2.14). This is a useful check, since there is no physical reason why anything untoward should happen at these special cases, even though, of course, none of them are close to being realized in our world. Additional checks are provided by the absence of poles $1 / \epsilon$ and $1 / \epsilon^{2}$, and by the cancellation ${ }^{5}$ of dependence on the Landau gauge Goldstone boson squared mass, after Goldstone boson resummation [13,14]. I have further checked that renormalization group invariance is satisfied

by Eqs. (2.1), (2.13), and (2.14), by computing the $Q$ derivative of each side using the known beta functions of the low-energy and high-energy theories and the direct $Q$ dependence of the function $\overline{\ln }(x)$. [Note that $F(x, y)$ has no $Q$ dependence.] In principle, this check should be merely equivalent to requiring the absence of poles in $\epsilon$, but in practice it also checks intermediate steps in the calculations.

\section{B. Matching of $\alpha_{S}$}

For the decoupling relation of $\alpha_{S}$, the result has already been obtained in pure QCD to one-loop [103], two-loop [104,105], three-loop [106], and four-loop order [108,109], and at complete two-loop order by Bednyakov [110]. I have recalculated the latter result, finding complete agreement:

$$
\theta_{\alpha_{S}}^{(1)}=\frac{2}{3} g_{3}^{2} \overline{\ln }(t)
$$

$$
\begin{aligned}
\theta_{\alpha_{S}}^{(2)}= & g_{3}^{4}\left[\frac{22}{9}+\frac{22}{3} \overline{\ln }(t)+\frac{4}{9} \overline{\ln }^{2}(t)\right]+g_{3}^{2} y_{t}^{2}\left\{\frac{2 t(h-t)}{h(4 t-h)^{2}} F(t, h)-\frac{2 t}{3 h}[1+\ln (h / t)]\right. \\
& +\frac{2 t}{3(4 t-Z)^{3}}\left[t\left(80 W / Z-7-64 W^{2} / Z^{2}\right)+8 Z-40 W+32 W^{2} / Z\right] F(t, Z) \\
& \left.+\left[\left(80 W / Z-64 W^{2} / Z^{2}\right)[1+\ln (Z / t)]+2+3 \overline{\ln }(h)-7 \overline{\ln }(Z)-14 \overline{\ln }(t)\right] / 18\right\} \\
& +g_{3}^{2} g^{2}\left\{\frac{8(W-Z)}{9 Z} \overline{\ln }(t)+3 \overline{\ln }(W)+\left(\frac{25 Z}{18 W}-\frac{13}{9}+\frac{14 W}{9 Z}\right) \overline{\ln }(Z)\right. \\
& \left.+\frac{t}{t-W} \ln (t / W)-\frac{49}{27}-\frac{W}{18 Z}-\frac{163 Z}{216 W}\right\}
\end{aligned}
$$

\footnotetext{
${ }^{5}$ More generally, in the Landau gauge tadpole-free scheme, this check is a nontrivial counterpart to the gauge-invariance check that one would obtain by instead working in a general gauge fixing with a tree-level VEV and nonvanishing tadpoles.
} 
Although equivalent, the presentation in Eq. (2.16) is somewhat more compact than the expression given in Ref. [110]. This is due in part to the use of the function $F(x, y)$ defined in Eq. (1.15) above, and also because the results are given in terms of running $\overline{\mathrm{MS}}$ squared masses here, rather than pole masses as in Ref. [110]; converting to the top quark pole mass in Eq. (2.15) just contributes some additional two-loop terms involving the one-loop top quark on-shell self-energy.

The pure QCD contributions to decoupling the top quark at three-loop [106] and four-loop [108,109] order are also reproduced here for the sake of completeness:

$\theta_{\alpha_{S}}^{(3)}=g_{3}^{6}\left[\frac{8}{27} \overline{\ln }^{3}(t)-3 \overline{\ln }^{2}(t)+\frac{620}{9} \overline{\ln }(t)+35.123151\right]$,

$$
\begin{aligned}
\theta_{\alpha_{S}}^{(4)}= & g_{3}^{8}\left[\frac{16}{81} \overline{\ln }^{4}(t)+\frac{4706}{81} \overline{\ln }^{3}(t)-\frac{1231}{27} \overline{\ln }^{2}(t)\right. \\
& +245.856958 \overline{\ln }(t)-109.765121] .
\end{aligned}
$$

The coefficients involving irrational numbers (available in their full glory in Refs. [106,108,109,133]) have been reduced to decimal approximations here and in similar expressions below, for the sake of brevity.

\section{Matching of running fermion masses}

Now, consider the decoupling relations for the masses of the fermions lighter than the top quark. The matching functions can be given generically for fermions other than the bottom quark, which is different because it has a direct coupling to the top quark and $W$ boson. For a generic fermion,

$$
\left(Q_{f}, I_{3}^{f}, C_{f}\right)= \begin{cases}(2 / 3,1 / 2,4 / 3) & (f=t, c, u), \\ (-1 / 3,-1 / 2,4 / 3) & (f=b, s, d), \\ (-1,-1 / 2,0) & (f=\tau, \mu, e)\end{cases}
$$

are the notations for electric charge $Q_{f}, I_{3}^{f}$ for the third component of weak isospin of the left-handed fermion, and $C_{f}$ for the $S U(3)_{c}$ Casimir invariant.

The method used is to require equality between two computations of the pole mass for each light fermion, first in the full standard model theory and then again in the lowenergy effective theory without $t, h, Z, W$. The strategy and details of the calculation of the light fermion pole masses that I have used are very similar to those described already in Ref. [80] for the top quark, and so they will not be reviewed here.

The resulting one-loop-order threshold corrections to the light fermion masses are

$\theta_{m_{f}}^{(1)}=\frac{9 g^{2}+3 g^{\prime 2}}{16}+Q_{f} g^{\prime 2}\left[I_{3}^{f}+Q_{f}(W / Z-1)\right][3 \overline{\ln }(Z)-5 / 2]$,

$\theta_{m_{b}}^{(1)}=\theta_{m_{d}}^{(1)}+\frac{3}{4} y_{t}^{2}\left[\frac{5}{6}-\overline{\ln }(t)+\left(\frac{W}{t-W}\right)^{2} \ln (t / W)-\frac{W}{t-W}\right]$

for a generic fermion other than the bottom quark, and for the bottom quark, respectively. In the case of the bottom quark, only the leading order in an expansion in $y_{b}^{2}$ has been kept. The next term in the expansion is

$$
\begin{aligned}
\Delta \theta_{m_{b}}^{(1)}= & y_{b}^{2}\left[\frac{3}{4} \overline{\ln }(h)+\frac{1}{4} \overline{\ln }(t)+\frac{W^{2}\left(3 t^{2}+4 t W-W^{2}\right)}{4(t-W)^{4}} \ln (t / W)-\frac{W^{2}(7 t-W)}{4(t-W)^{3}}+\frac{7 Z^{2}+16 W Z-32 W^{2}}{36 Z^{2}} \overline{\ln }(Z)\right. \\
& \left.-\frac{4(Z-W)(2 W+Z)}{9 Z^{2}} \overline{\ln }(b)-\frac{91}{216}-\frac{8 W}{27 Z}+\frac{16 W^{2}}{27 Z^{2}}\right] .
\end{aligned}
$$

However, since $y_{b}^{2} / 16 \pi^{2}<2 \times 10^{-6}$, this contribution is negligible.

The two-loop-order threshold function for the bottom quark mass takes the form

$$
\begin{aligned}
\theta_{m_{b}}^{(2)}= & \frac{4}{3} g_{3}^{4}\left[\overline{\ln }^{2}(t)+\frac{5}{3} \overline{\ln }(t)+\frac{89}{36}\right]+g_{3}^{2} y_{t}^{2}\left[\left(8 t^{2}-8 t W+6 W^{2}\right) I(0, t, W)+t\left(7 t^{2}-17 t W+22 W^{2}\right) \overline{\ln }^{2}(t)\right. \\
& +2 t W(4 t-7 W) \overline{\ln }(t) \overline{\ln }(W)+\left(35 t^{2} W-23 t^{3}-56 t W^{2}+16 W^{3}\right) \overline{\ln }(t)-(2 t-3 W)(7 t-3 W) W \overline{\ln }(W) \\
& \left.+92 t^{3} / 3-19 t^{2} W+17 t W^{2}+4 W^{3} / 3\right] /(t-W)^{3}+\frac{4}{3} g_{3}^{2}\left\{\frac{g^{2}}{6}(1+2 W / Z)[\overline{\ln }(Z)-17 / 12]+\frac{9}{4} g^{2} \overline{\ln }(W)\right. \\
& \left.+\frac{9}{8}\left(g^{2}+g^{\prime 2}\right) \overline{\ln }(Z)-\frac{15}{32}\left(3 g^{2}+g^{\prime 2}\right)\right\}+\sum_{j=1}^{12} b_{j}^{(2)} \mathcal{I}_{j}^{(2)}+\sum_{j=1}^{4} \sum_{k=1}^{j} b_{j, k}^{(1,1)} \mathcal{I}_{j}^{(1)} \mathcal{I}_{k}^{(1)}+\sum_{j=1}^{4} b_{j}^{(1)} \mathcal{I}_{j}^{(1)}+b^{(0)} .
\end{aligned}
$$


The part that does not contain the strong coupling $g_{3}$ involves coefficients of two-loop integral functions and logarithms from the lists

$$
\begin{aligned}
\mathcal{I}^{(2)}= & \left\{\zeta_{2}, I(0, h, W), I(0, h, Z), I(0, W, Z), I(h, W, W), I(h, Z, Z),\right. \\
& I(t, t, Z), I(W, W, Z), I(0, t, W), I(h, t, t), I(h, t, W), I(t, W, Z)\},
\end{aligned}
$$

$$
\mathcal{I}^{(1)}=\{\overline{\ln }(t), \overline{\ln }(h), \overline{\ln }(Z), \overline{\ln }(W)\},
$$

respectively. It cannot be simplified to a length reasonable for printing, and so it is not given explicitly above in its full form, but instead in an ancillary electronic file distributed with this paper, called theta2mb. The file theta2mb contains the coefficients in Eq. (2.23). The individual coefficients $b_{j}^{(2)}, b_{j, k}^{(1,1)}, b_{j}^{(1)}$, and $b^{(0)}$ are rational functions of the input parameters $t, h, Z, W$, and $v$. Many of them have poles in one or more of the quantities $t-W, 4 W-h$, $4 Z-h, 4 t-Z, \lambda(t, W, Z)$, and $\lambda(t, W, h)$, but I have checked that the total $\theta_{m_{b}}^{(2)}$ is nevertheless finite when each of these quantities vanishes. The format used in the ancillary file theta $2 \mathrm{mb}$ is compatible with inclusion in computer code for easy numerical evaluation using Eqs. (1.9)-(1.15). Additional checks follow, as usual, from the absence of poles $1 / \epsilon^{2}$ and $1 / \epsilon$ upon translating to the $\overline{\mathrm{MS}}$ scheme, and by the cancellation of contributions involving the Landau gauge Goldstone boson mass.

For generic fermions $f=(c, s, u, d, \tau, \mu, e)$, the twoloop threshold functions are similarly found to be

$$
\begin{aligned}
\theta_{m_{f}}^{(2)}= & C_{f} g_{3}^{4}\left[\overline{\ln ^{2}}(t)+\frac{5}{3} \overline{\ln }(t)+\frac{89}{36}\right]+C_{f} g_{3}^{2}\left\{3 g ^ { \prime 2 } Q _ { f } \left[I_{3}^{f}\right.\right. \\
& \left.+Q_{f}(W / Z-1)\right][\overline{\ln }(Z)-17 / 12] \\
& \left.+\frac{9}{4} g^{2} \overline{\ln }(W)+\frac{9}{8}\left(g^{2}+g^{\prime 2}\right) \overline{\ln }(Z)-\frac{15}{32}\left(3 g^{2}+g^{\prime 2}\right)\right\} \\
& +\sum_{j=1}^{8} c_{j}^{(2)} \mathcal{I}_{j}^{(2)}+\sum_{j=1}^{4} \sum_{k=1}^{j} c_{j, k}^{(1,1)} \mathcal{I}_{j}^{(1)} \mathcal{I}_{k}^{(1)} \\
& +\sum_{j=1}^{4} c_{j}^{(1)} \mathcal{I}_{j}^{(1)}+c^{(0)},
\end{aligned}
$$

where the contributions independent of $g_{3}$ involve coefficients that are again too complicated to show in print, and so are relegated to an electronic file called theta2mf distributed as an ancillary to this paper. The file theta $2 \mathrm{mf}$ contains the coefficients in Eq. (2.26). Note that the last four functions in the list in Eq. (2.24) do not appear in Eq. (2.26). The individual coefficients $c_{j}^{(2)}, c_{j, k}^{(1,1)}, c_{j}^{(1)}$, and $c^{(0)}$ are again rational functions of $t, h, Z, W$, and $v$, with pole singularities at $4 Z-h$ and $4 t-Z$, but the total is free of these singularities.

The pure QCD threshold corrections for light quark masses were already known up to three-loop order from
Chetyrkin, Kniehl, and Steinhauser in Ref. [106] and from Liu and Steinhauser at four-loop order in Ref. [111]. They are listed here for the sake of completeness. For each quark $q=(b, c, s, u, d)$,

$$
\begin{aligned}
\theta_{m_{q}}^{(3)}= & g_{3}^{6}\left[-\frac{152}{27} \overline{\ln }^{3}(t)+\frac{700}{27} \overline{\ln }^{2}(t)\right. \\
& +111.047973 \overline{\ln }(t)+126.160947],
\end{aligned}
$$

$$
\begin{aligned}
\theta_{m_{q}}^{(4)}= & g_{3}^{8}\left[\frac{830}{27} \overline{\ln }^{4}(t)-\frac{10984}{81} \overline{\ln }^{3}(t)-543.379386 \overline{\ln }^{2}(t)\right. \\
& +452.388432 \overline{\ln }(t)+236.908052]
\end{aligned}
$$

Note that the preceding equations apply specifically to the decoupling of the top quark from the theory. Again, the known irrational parts have been replaced by decimal approximations.

\section{DECOUPLING OF LIGHTER FERMIONS IN THE QCD + QED EFFECTIVE THEORY}

In this section, I provide the decoupling relations appropriate for further sequential decoupling of fermions within the QCD + QED theory. None of the results in this section are new, as the QCD parts of these are now well known, and the QED contributions at up to two-loop order and certain light mass expansions can be easily inferred from those found in the existing literature. They are collected here for the sake of completeness.

The notation adopted here assumes that a generic fermion, denoted $F$, is to be decoupled. ${ }^{6}$ The charge and QCD Casimir quantum numbers of $F$ are to be denoted $Q_{F}$ and $C_{F}$, respectively, just as in Eq. (2.19), and the index $T_{F}$ equals $1 / 2$ when the decoupled fermion $F$ is a quark and 0 when it is a lepton, while the number of colors $N_{F}$ is 3 when $F$ is a quark and 1 when $F$ is a lepton. The decoupling scale $Q$ associated with the matching of parameters is again arbitrary, but typically should be chosen to be comparable to the mass of $F$, in order to avoid large logarithms in

\footnotetext{
${ }^{6}$ In the standard model, the formulas below are not practically applicable with $F=u, d$, s, because QCD perturbation theory is not under control. Instead, the RPP [126] quotes the $\overline{\mathrm{MS}}$ masses at $Q=2 \mathrm{GeV}$.
} 
observables calculated after using the renormalization group equations to run the surviving parameters to lower energies. The running $\overline{\mathrm{MS}}$ parameters of the high-energy $S U(3)_{c} \times U(1)_{\mathrm{EM}}$ theory will be denoted $\alpha, \alpha_{S}, F \equiv m_{F}^{2}$, and $m_{f}$, where $f$ runs over the list of the lighter fermions which are not being decoupled. For the low-energy theory with $F$ decoupled, the parameters are distinguished by an underline, so they are $\underline{\alpha}, \underline{\alpha}_{S}$, and $\underline{m}_{f}$. The number of light quark flavors among the fermions $f$ in the decoupled theory (which will also include leptons) will be denoted $n_{q}$.

The decoupling relations can then be written in the form

$$
\begin{array}{r}
\alpha(Q)=\alpha(Q)\left[1+\sum_{\ell=1}^{\infty} \frac{1}{(4 \pi)^{\ell}} \vartheta_{\alpha}^{(\ell)}\right], \\
\underline{\alpha}_{S}(Q)=\alpha_{S}(Q)\left[1+\sum_{\ell=1}^{\infty} \frac{1}{(4 \pi)^{\ell}} \vartheta_{\alpha_{S}}^{(\ell)}\right], \\
\underline{m}_{f}(Q)=m_{f}(Q)\left[1+\sum_{\ell=1}^{\infty} \frac{1}{(4 \pi)^{\ell}} \vartheta_{m_{f}}^{(\ell)}\right] \quad(f \neq F) .
\end{array}
$$

(Note that the symbol $\vartheta$ is used to denote the threshold corrections within the QCD + QED theory in this section, in distinction from the symbol $\theta$ used in the previous section for decoupling $t, h, Z, W$.) Then, for the matching coefficients for the electromagnetic coupling, one has at the scale $Q$ where $F$ is decoupled

$$
\begin{gathered}
\vartheta_{\alpha}^{(1)}=\frac{4}{3} N_{F} Q_{F}^{2} \alpha \overline{\ln }(F), \\
\vartheta_{\alpha}^{(2)}=\left[\frac{4}{3} N_{F} Q_{F}^{2} \alpha \overline{\ln }(F)\right]^{2}-N_{F} Q_{F}^{2} \alpha\left(C_{F} \alpha_{S}\right. \\
\left.+Q_{F}^{2} \alpha\right)\left[4 \overline{\ln }(F)+\frac{13}{3}\right] .
\end{gathered}
$$

For the QCD coupling, the results through two-loop order including QED effects are

$$
\begin{gathered}
\vartheta_{\alpha_{S}}^{(1)}=\frac{4}{3} T_{F} \alpha_{S} \overline{\ln }(F) \\
\vartheta_{\alpha_{S}}^{(2)}=\left[\frac{4}{3} T_{F} \alpha_{S} \overline{\ln }(F)\right]^{2}-T_{F} \alpha_{S}\left(C_{F} \alpha_{S}+Q_{F}^{2} \alpha\right)\left[4 \overline{\ln }(F)+\frac{13}{3}\right] \\
+T_{F} C_{A} \alpha_{S}^{2}\left[\frac{20}{3} \overline{\ln }(F)+\frac{32}{9}\right]
\end{gathered}
$$

where $T_{F}=1 / 2$ when $F$ is a quark, and $T_{F}=0$ when $F$ is a lepton, and $C_{A}=3$. The pure QCD contributions at threeloop and four-loop order, which apply only if $F$ is a quark, are found from Refs. [106,108,109]:

$$
\begin{aligned}
\vartheta_{\alpha_{S}}^{(3)}= & \alpha_{S}^{3}\left[\frac{8}{27} \overline{\ln }^{3}(F)+\left(\frac{53}{9}-\frac{16}{9} n_{q}\right) \overline{\ln }^{2}(F)\right. \\
& \left.+\left(\frac{955}{9}-\frac{67}{9} n_{q}\right) \overline{\ln }(F)+62.211628-\frac{2633}{486} n_{q}\right] \\
\vartheta_{\alpha_{S}}^{(4)}= & \alpha_{S}^{4}\left[\frac{16}{81} \overline{\ln }^{4}(F)+\left(\frac{3766}{81}+\frac{508}{81} n_{q}-\frac{64}{81} n_{q}^{2}\right) \overline{\ln }^{3}(F)\right. \\
& +\left(\frac{4354}{27}-\frac{2966}{81} n_{q}-\frac{77}{81} n_{q}^{2}\right) \overline{\ln }^{2}(F) \\
& +\left(2157.863053-335.316171 n_{q}-\frac{6865}{729} n_{q}^{2}\right) \overline{\ln }(F) \\
& \left.+1323.608830-258.542470 n_{q}-5.626464 n_{q}^{2}\right]
\end{aligned}
$$

These can be used with $n_{q}=4$ when $F$ is the bottom quark, and with $n_{q}=3$ when $F$ is the charm quark. The formulas with $n_{q}=5$, of course, coincide with that for decoupling the top quark, as in Eqs. (2.27) and (2.28) above.

The one-loop and two-loop threshold corrections for each light fermion mass $m_{f}$ when decoupling the fermion $F$ in the $S U(3)_{c} \times U(1)_{\mathrm{EM}}$ theory are

$$
\begin{gathered}
\vartheta_{m_{f}}^{(1)}=0 \\
\vartheta_{m_{f}}^{(2)}=2\left(T_{F} C_{f} \alpha_{S}^{2}+N_{F} Q_{F}^{2} Q_{f}^{2} \alpha^{2}\right)\left[\overline{\ln }^{2}(F)+\frac{5}{3} \overline{\ln }(F)\right. \\
\left.+\frac{89}{36}+\Delta_{2}(f / F)\right],
\end{gathered}
$$

where the last term is the power-suppressed mass correction, with $f, F$ being the $\overline{\mathrm{MS}}$ squared masses and

$\Delta_{2}(r)=r\left(\frac{8}{15} \ln (r)-\frac{76}{75}\right)+r^{2}\left(\frac{9}{70} \ln (r)-\frac{1389}{9800}\right)+\mathcal{O}\left(r^{3}\right)$.

This effect is mentioned because the squared mass ratios occurring in the decoupling of the light fermions (notably, $c / b \sim 0.1)$ are not quite as suppressed as $b / t$ in the decoupling of the top quark in the previous section, but its numerical impact is still quite small. It can be obtained from the two-loop result for a quark pole mass in the presence of other massive and massless quarks in Ref. [60]. The pure QCD corrections are also known at three-loop and four-loop orders from Refs. [106,111], respectively: 


$$
\begin{gathered}
\vartheta_{m_{f}}^{(3)}=\alpha_{S}^{3}\left[\left(\frac{16}{27} n_{q}-\frac{232}{27}\right) \overline{\ln }^{3}(F)+\frac{700}{27} \overline{\ln }^{2}(F)+\left(\frac{212}{27} n_{q}+71.788714\right) \overline{\ln }(F)\right. \\
\left.+118.248112+1.582567 n_{q}+\Delta_{3}(f / F)\right] \\
\vartheta_{m_{f}}^{(4)}=\alpha_{S}^{4}\left[\left(\frac{8}{27} n_{q}^{2}-\frac{80}{9} n_{q}+\frac{610}{9}\right) \overline{\ln }^{4}(F)+\left(\frac{184}{9} n_{q}-\frac{19264}{81}\right) \overline{\ln }^{3}(F)+\left(\frac{496}{81} n_{q}^{2}-\frac{15650}{81} n_{q}+269.583577\right) \overline{\ln }^{2}(F)\right. \\
\left.+\left(286.364218+39.625147 n_{q}-1.284061 n_{q}^{2}\right) \overline{\ln }(F)+14.375890 n_{q}^{2}-375.221169 n_{q}+1753.616640\right] .
\end{gathered}
$$

In the three-loop part, the small mass correction is

$$
\begin{aligned}
\Delta_{3}(r)= & \frac{8}{9}\left(2 n_{q}-31\right) \overline{\ln }(F) \Delta_{2}(r)+r\left\{\left(\frac{64}{135} n_{q}-\frac{451}{81}\right) \ln ^{2}(r)+\left(\frac{84887}{7290}-\frac{128}{135} n_{q}\right) \ln (r)+2.77670-0.22452 n_{q}\right\} \\
& +r^{2}\left\{\left(\frac{4}{35} n_{q}-\frac{239}{270}\right) \ln ^{2}(r)+\left(\frac{580157}{396900}-\frac{6}{35} n_{q}\right) \ln (r)+0.52092+0.03556 n_{q}\right\}+\mathcal{O}\left(r^{3}\right),
\end{aligned}
$$

which can be gleaned from the expansion of the pole mass given in Ref. [131] based on the results in Refs. [134,135]. In the four-loop part, the expansion is not known beyond the lowest order in $r=f / F$.

In applications of the above formulas, the renormalization group running between scales requires the beta functions for the two gauge couplings and the fermion masses, which are known in the $S U(3)_{c} \times U(1)_{\mathrm{EM}}$ theory at full three-loop order including electromagnetic effects; see, for example, Ref. [131] (and the Appendix of Ref. [136]) for a general product gauge group with an arbitrary reducible fermion representation. The higherorder QCD corrections to the beta function for $\alpha$ are given in Ref. [123] at order $\alpha^{2} \alpha_{S}^{3}$ and in Ref. [137] at order $\alpha^{2} \alpha_{S}^{4}$. The four-loop and five-loop pure QCD contributions to the $\alpha_{S}$ beta function are found in Refs. [95,96] and [97,98], respectively. The three-loop, four-loop and five-loop pure QCD contributions to the quark mass beta functions are in Refs. [99-102]. Also useful in this context are the fermion pole masses, which are given for a general product gauge group with an arbitrary reducible fermion representation (but assuming just one nonzero fermion mass) in the Appendix of Ref. [136], with four-loop pure QCD contributions in Refs. [64,65]. In the case of more than one nonzero quark mass, expansions for small and large mass ratios in the three-loop pole masses have been given in Refs. [134,131].

\section{NUMERICAL RESULTS}

In this section, I will illustrate the numerical impact of the matching conditions, concentrating on the new results of this paper-i.e., the shifts in the electromagnetic coupling and the light fermion masses from decoupling $t, h, Z, W$ in the standard model, as a function of the matching scale $Q$. For a benchmark model, I consider the following numerical values for standard model parameters at a reference scale $Q_{0}=173.34 \mathrm{GeV}$ :

$$
\begin{gathered}
g_{3}=1.1666, \\
g=0.647550, \\
g^{\prime}=0.358521, \\
y_{t}=0.93690, \\
\lambda=0.12597, \\
v=246.647 \mathrm{GeV} .
\end{gathered}
$$

These are then run to a matching scale $80 \mathrm{GeV}<$ $Q<180 \mathrm{GeV}$, and the figures below show the resulting matching corrections obtained in Secs. II A and II C.

First, Fig. 2 shows results for the various contributions to the fractional shift in $\alpha$ :

$$
\delta \alpha / \alpha \equiv \frac{1}{16 \pi^{2}} \theta_{\alpha}^{(1)}+\frac{1}{\left(16 \pi^{2}\right)^{2}} \theta_{\alpha}^{(2)}+\cdots .
$$

The left panel of Fig. 2 shows the dominant one-loop contribution from Eq. (2.13), as well as the total from Eqs. (2.13) and (2.14). The right panel shows the breakdown of the two-loop contribution in Eq. (2.14) into the part proportional to $g_{3}^{2}$, the part proportional to $y_{t}^{2}$, the remaining pure electroweak part, and the total of these twoloop corrections. As might be expected, the pure 

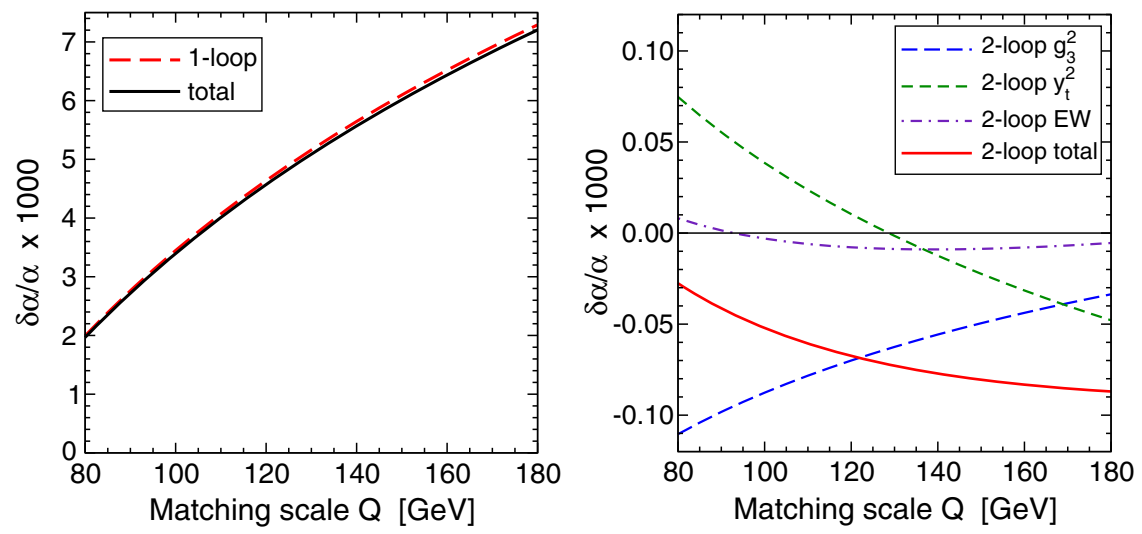

FIG. 2. Contributions to the matching relation fractional shift in $\alpha$ from decoupling $t, h, Z, W$ in the standard model, as a function of the matching renormalization scale $Q$. The left panel shows the dominant one-loop contribution (dashed line) from Eq. (2.13), as well as the total (solid line). The right panel shows the breakdown of the total two-loop contribution from Eq. (2.14) (solid line) into the part proportional to $g_{3}^{2}$ (long-dashed line), the part proportional to $y_{t}^{2}$ (short-dashed line), and the remaining electroweak part (dot-dashed line).

electroweak two-loop contributions are quite small over the entire range of $Q$, never exceeding 1 part in $10^{5}$. The twoloop $g_{3}^{2}$ and $y_{t}^{2}$ parts are larger, but for lower $Q$ there is significant cancellation between them. The total two-loop contribution ranges from about $-3 \times 10^{-5}$ to $-9 \times 10^{-5}$, depending on the choice of $Q$. This is comparable to the present uncertainty on $\Delta \alpha_{\text {had }}^{(5)}\left(m_{Z}\right)$ estimated in the RPP [126], which is $7 \times 10^{-5}$. Therefore, the total two-loop correction is just barely numerically relevant at the present time. If improvements in the hadronic uncertainty are forthcoming, then the two-loop corrections will become correspondingly more significant. However, it seems unlikely that further three-loop corrections to the matching of $\alpha$ from decoupling $t, h, Z, W$ will be needed in the foreseeable future.

The fractional shifts

$$
\delta m_{f} / m_{f} \equiv \frac{1}{16 \pi^{2}} \theta_{m_{f}}^{(1)}+\frac{1}{\left(16 \pi^{2}\right)^{2}} \theta_{m_{f}}^{(2)}+\cdots
$$

are shown in Figs. 3-5. For each of the quark masses, the solid line is the total matching fractional shift, and the separate contributions from one-loop (to which QCD does not contribute) and the combined two-, three-, and fourloop QCD contributions are shown as the long-dashed and short-dashed lines, respectively. In the case of the bottom quark as shown in Fig. 3, the remaining two-loop mixed QCD and non-QCD contributions are each comparable in magnitude to the three-loop pure QCD part and much larger than the four-loop pure QCD part (not shown separately), but they have opposite signs from each other and have a significant cancellation. The total fractional shift in $m_{b}$ from decoupling $t, h, Z, W$ is always less than $5 \times 10^{-3}$, and it happens to be very small for $Q$ near $M_{Z}$ due to accidental cancellation between the different contributions.
(A similar numerical study of the threshold correction for $m_{b}$ was conducted in Ref. [131], but with different details, because that reference uses a different definition of the high-energy running bottom quark mass, based on the VEV definition $v_{\text {on-shell }}^{2}=1 / \sqrt{2} G_{F}$.)

In Fig. 4, the results for the down and strange quark masses are shown in the left panel, and those for the charm and up quark masses are shown in the right panel. In both cases, the two-loop non-QCD corrections are quite tiny, in part because there is no $y_{t}$ enhancement as there was for the bottom quark. The two-loop mixed QCD corrections are larger in magnitude than the four-loop ones and are comparable to the three-loop QCD corrections, but still

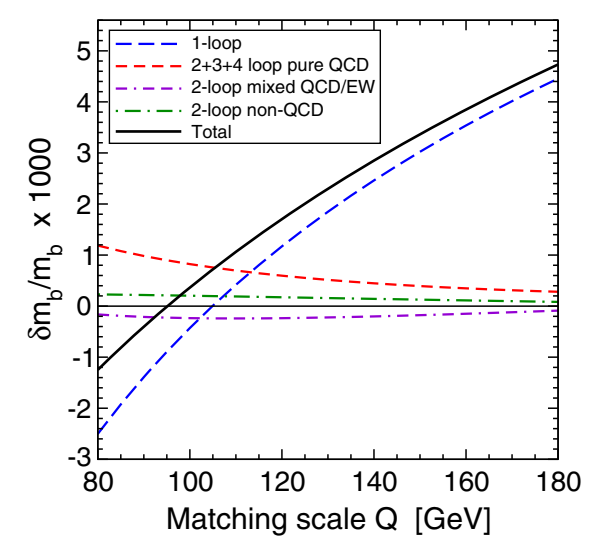

FIG. 3. Contributions to the matching relation fractional shift in the $\overline{\mathrm{MS}}$ bottom quark mass from decoupling $t, h, Z, W$ in the standard model, as a function of the matching renormalization scale $Q$. The long-dashed line is the one-loop contribution from Eq. (2.21). The short-dashed line is the total QCD (two-, three-, and four-loop) contribution, from the $g_{3}^{4}$ part of Eq. (2.23) and Eqs. (2.27) and (2.28). The lower and upper dash-dotted lines are from the $g_{3}^{2}$ (mixed QCD) and $g_{3}^{0}$ (non-QCD) parts of Eq. (2.23), respectively. The solid line is the total. 

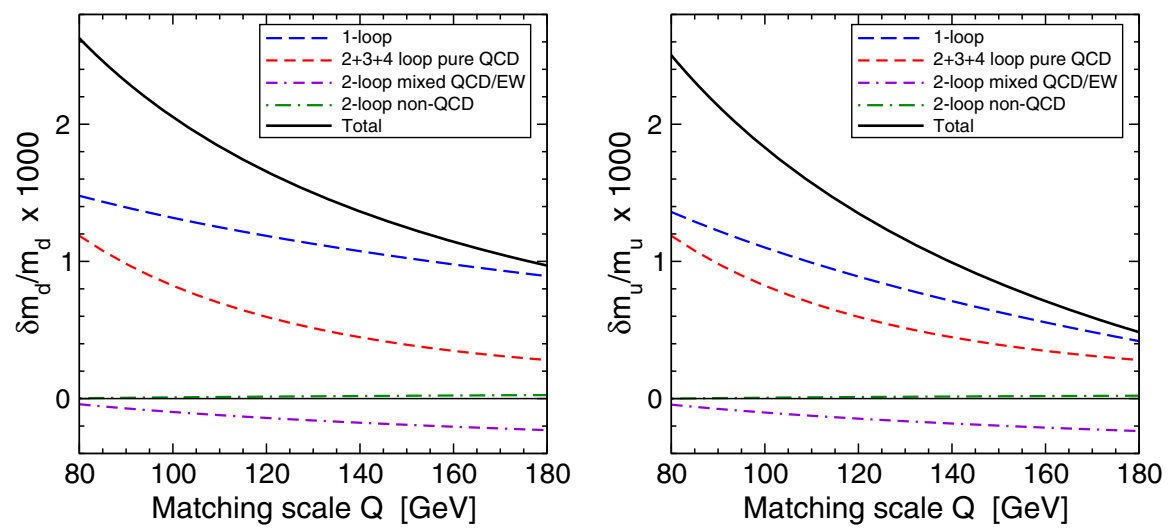

FIG. 4. Contributions to the matching relation fractional shift in the $\overline{\mathrm{MS}}$ quark masses from decoupling $t, h, Z, W$ in the standard model, as a function of the matching renormalization scale $Q$. The left panel shows $\delta m_{s} / m_{s}=\delta m_{d} / m_{d}$, and the right panel shows $\delta m_{c} / m_{c}=\delta m_{u} / m_{u}$. In each case, the long-dashed line is the one-loop contribution from Eq. (2.20). The short-dashed line is the total QCD (two-, three-, and four-loop) contribution, from the $g_{3}^{4}$ part of Eq. (2.26) and Eqs. (2.27) and (2.28), and the lower and upper dashdotted lines are from the $g_{3}^{2}$ (mixed QCD) and $g_{3}^{0}$ (non-QCD) parts of Eq. (2.26), respectively. The solid line is the total.

less than $2 \times 10^{-4}$ over most of the range of choices of $Q$. For each of the $c, s, u, d$ quark masses, the total fractional shifts are slightly larger than $2 \times 10^{-3}$ for $Q$ near $M_{Z}$, and they decrease with increasing $Q$. So, they are considerably smaller than the present experimental uncertainties in the masses. This situation is likely to persist for some time, pending dramatic improvements in the low-energy $\overline{\mathrm{MS}}$ quark mass determinations from, e.g., lattice QCD.

Figure 5 shows the results for the charged lepton $(\tau, \mu, e)$ masses, for which there are, of course, no QCD-enhanced corrections through two-loop order. As expected, the matching is dominated by the one-loop part, which contributes on the order of $2 \times 10^{-4}$ to $2 \times 10^{-3}$ to $\delta m_{e} / m_{e}=\delta m_{\mu} / m_{\mu}=$ $\delta m_{\tau} / m_{\tau}$, depending on the choice of matching scale $Q$. The two-loop contribution to the fractional matching shift is seen

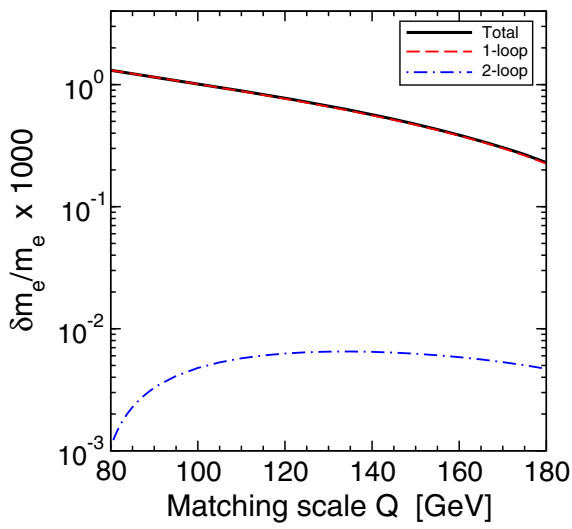

FIG. 5. Contributions to the matching relation fractional shift in the $\overline{\mathrm{MS}}$ charged lepton masses from decoupling $t, h, Z, W$ in the standard model, as a function of the matching renormalization scale $Q$. The solid line is the total, and the long-dashed line hiding just beneath it is the dominant one-loop contribution from Eq. (2.20). The small difference is the two-loop contribution from Eq. (2.26), shown as the dot-dashed line. to be always less than $6 \times 10^{-6}$. This can be compared to the fractional experimental uncertainty in the physical masses of the charged leptons from Ref. [126]. For the tau lepton, this is presently about $7 \times 10^{-5}$, showing that the two-loop contribution is already safely smaller than the accuracy needed under the most optimistic of circumstances. For the muon, the fractional uncertainty in the physical mass is about $2 \times 10^{-8}$, and for the electron is about $6 \times 10^{-9}$, so in those cases the two-loop (and perhaps even higher-loop) threshold matching contributions are worthwhile, at least in principle. However, this does not yet take into account more subtle parametric uncertainties that are beyond the scope of this paper-for example, the low-energy nonperturbative hadronic contribution to their pole masses induced through photon self-energy corrections, and even small loop effects from $G_{F}$-suppressed four-fermion couplings in the lowenergy effective field theory.

\section{OUTLOOK}

In this paper, I have discussed the matching relations for the renormalizable couplings in the low-energy effective $S U(3)_{c} \times U(1)_{\mathrm{EM}}$ gauge theory with five quarks and three leptons, when the top quark, Higgs scalar, and $Z$ and $W$ vector bosons are decoupled together at an $\overline{\mathrm{MS}}$ renormalization scale $Q$. This simultaneous decoupling ensures that the low-energy effective field theory has marginal and relevant couplings as part of a consistent renormalizable gauge theory. Also present in the low-energy theory are nonrenormalizable couplings including four-fermion terms for the effective weak interactions; the matching relations for those are not discussed in the present paper. The matching relations provide a connection to the far ultraviolet, fundamental, and complete definition of the standard model. The new results for the matching of the electromagnetic coupling $\alpha$ and the light quark and lepton masses 
augment previously known results for the strong coupling and the bottom quark mass, and the latter is given here in the tadpole-free scheme for the VEV, as part of a larger program [11-13,22,57,58,80] to relate standard model observables to the underlying Lagrangian parameters in that scheme. The matching corrections found here are reassuringly small, and in some cases much smaller than the present experimental uncertainties in the corresponding observables. They nevertheless are at least useful in providing informed bounds on the possible sources of theoretical error. They could become considerably more significant in the future when experimental uncertainties on input parameters, notably the low-energy quark masses and nonperturbative contributions to the fine-structure constant, are reduced.

\section{ACKNOWLEDGMENTS}

I am grateful to Jens Erler and Ayres Freitas for helpful communications regarding Ref. [126]. This work was supported in part by National Science Foundation Grant No. PHY-1719273.
[1] W. A. Bardeen, A. J. Buras, D. W. Duke, and T. Muta, Deep inelastic scattering beyond the leading order in asymptotically free gauge theories, Phys. Rev. D 18, 3998 (1978).

[2] E. Braaten and J.P. Leveille, Minimal subtraction and momentum subtraction in QCD at two loop order, Phys. Rev. D 24, 1369 (1981).

[3] C. G. Bollini and J. J. Giambiagi, Lowest order divergent graphs in $\nu$-dimensional space, Phys. Lett. 40B, 566 (1972).

[4] J. F. Ashmore, A method of gauge invariant regularization, Lett. Nuovo Cimento 4, 289 (1972).

[5] G. M. Cicuta and E. Montaldi, Analytic renormalization via continuous space dimension, Lett. Nuovo Cimento 4, 329 (1972).

[6] G. 't Hooft and M. J. G. Veltman, Regularization and renormalization of gauge fields, Nucl. Phys. B44, 189 (1972).

[7] G. 't Hooft, Dimensional regularization and the renormalization group, Nucl. Phys. B61, 455 (1973).

[8] C. Ford, I. Jack, and D. R. T. Jones, The standard model effective potential at two loops, Nucl. Phys. B387, 373 (1992); Erratum, Nucl. Phys. B504, 551 (1997); The effective potential and the differential equations method for Feynman integrals, Phys. Lett. B 274, 409 (1992); Erratum, Phys. Lett. B 285, 399 (1992).

[9] S. P. Martin, Two loop effective potential for a general renormalizable theory and softly broken supersymmetry, Phys. Rev. D 65, 116003 (2002).

[10] S. P. Martin, Three-loop standard model effective potential at leading order in strong and top Yukawa couplings, Phys. Rev. D 89, 013003 (2014).

[11] S. P. Martin, Effective potential at three loops, Phys. Rev. D 96, 096005 (2017).

[12] S. P. Martin, Four-loop standard model effective potential at leading order in QCD, Phys. Rev. D 92, 054029 (2015).

[13] S. P. Martin, Taming the Goldstone contributions to the effective potential, Phys. Rev. D 90, 016013 (2014).

[14] J. Elias-Miro, J. R. Espinosa, and T. Konstandin, Taming infrared divergences in the effective potential, J. High Energy Phys. 08 (2014) 034.
[15] S. P. Martin and D. G. Robertson, Evaluation of the general 3-loop vacuum Feynman integral, Phys. Rev. D 95, 016008 (2017); the software package 3VIL (3-loop Vacuum Integral Library) implementing these results is available from http://www.niu.edu/spmartin/3VIL/.

[16] A. Freitas, Three-loop vacuum integrals with arbitrary masses, J. High Energy Phys. 11 (2016) 145; S. Bauberger and A. Freitas, TVID: Three-loop vacuum integrals from dispersion relations, arXiv:1702.02996.

[17] See, for example, J. Fleischer and F. Jegerlehner, Radiative corrections to Higgs decays in the extended WeinbergSalam model, Phys. Rev. D 23, 2001 (1981).

[18] S. P. Martin and H. H. Patel, Two-loop effective potential for generalized gauge fixing, Phys. Rev. D 98, 076008 (2018).

[19] F. Bezrukov, M. Y. Kalmykov, B. A. Kniehl, and M. Shaposhnikov, Higgs boson mass and new physics, J. High Energy Phys. 10 (2012) 140.

[20] G. Degrassi, S. Di Vita, J. Elias-Miro, J. R. Espinosa, G. F. Giudice, G. Isidori, and A. Strumia, Higgs mass and vacuum stability in the standard model at NNLO, J. High Energy Phys. 08 (2012) 098.

[21] D. Buttazzo, G. Degrassi, P. P. Giardino, G. F. Giudice, F. Sala, A. Salvio, and A. Strumia, Investigating the nearcriticality of the Higgs boson, J. High Energy Phys. 12 (2013) 089.

[22] S. P. Martin and D. G. Robertson, Higgs boson mass in the standard model at two-loop order and beyond, Phys. Rev. D 90, 073010 (2014).

[23] B. A. Kniehl, A. F. Pikelner, and O. L. Veretin, Two-loop electroweak threshold corrections in the standard model, Nucl. Phys. B896, 19 (2015).

[24] B. A. Kniehl, A. F. Pikelner, and O. L. Veretin, mr: A C++ library for the matching and running of the standard model parameters, Comput. Phys. Commun. 206, 84 (2016).

[25] A. Sirlin, Radiative corrections in the $S U(2)_{L} \times U(1)$ theory: A simple renormalization framework, Phys. Rev. D 22, 971 (1980).

[26] W. J. Marciano and A. Sirlin, Radiative corrections to neutrino induced neutral current phenomena in the $S U(2)_{L} \times U(1)$ theory, Phys. Rev. D 22, 2695 (1980); Erratum, Phys. Rev. D 31, 213 (1985). 
[27] W. J. Marciano and A. Sirlin, Testing the standard model by precise determinations of $W^{ \pm}$and $Z$ masses, Phys. Rev. D 29, 945 (1984); Erratum, Phys. Rev. D 31, 213 (1985).

[28] A. Sirlin, On the $\mathrm{O}\left(\alpha^{2}\right)$ corrections to $\tau_{\mu}, m_{W}, m_{Z}$ in the $S U(2)_{L} \times U(1)$ theory, Phys. Rev. D 29, 89 (1984).

[29] A. Djouadi and C. Verzegnassi, Virtual very heavy top effects in LEP/SLC precision measurements, Phys. Lett. B 195, 265 (1987).

[30] A. Djouadi, $\mathcal{O}\left(\alpha \alpha_{S}\right)$ vacuum polarization functions of the standard model gauge bosons, Nuovo Cimento A 100, 357 (1988).

[31] M. Consoli, W. Hollik, and F. Jegerlehner, The effect of the top quark on the $M_{W}-M_{Z}$ interdependence and possible decoupling of heavy fermions from low-energy physics, Phys. Lett. B 227, 167 (1989).

[32] B. A. Kniehl, Two loop corrections to the vacuum polarizations in perturbative QCD, Nucl. Phys. B347, 86 (1990).

[33] F. Halzen and B. A. Kniehl, $\Delta r$ beyond one loop, Nucl. Phys. B353, 567 (1991).

[34] A. Djouadi and P. Gambino, Electroweak gauge boson self-energies: Complete QCD corrections, Phys. Rev. D 49, 3499 (1994); Erratum, Phys. Rev. D 53, 4111 (1996).

[35] L. Avdeev, J. Fleischer, S. Mikhailov, and O. Tarasov, $\mathcal{O}\left(\alpha \alpha_{S}^{2}\right)$ correction to the electroweak $\rho$ parameter, Phys. Lett. B 336, 560 (1994); Erratum, Phys. Lett. B 349, 597 (1995).

[36] K. G. Chetyrkin, J. H. Kühn, and M. Steinhauser, Corrections of order $\mathcal{O}\left(G_{F} M_{t}^{2} \alpha_{S}^{2}\right)$ to the $\rho$ parameter, Phys. Lett. B 351, 331 (1995).

[37] K. G. Chetyrkin, J. H. Kühn, and M. Steinhauser, QCD Corrections from Top Quark to Relations Between Electroweak Parameters to Order $\alpha_{S}^{2}$, Phys. Rev. Lett. 75, 3394 (1995).

[38] G. Degrassi, P. Gambino, and A. Vicini, Two loop heavy top effects on the $m_{Z}-m_{W}$ interdependence, Phys. Lett. B 383, 219 (1996).

[39] G. Degrassi, P. Gambino, and A. Sirlin, Precise calculation of $M_{W}, \sin ^{2} \theta_{W}\left(M_{Z}\right)$, and $\sin ^{2} \theta_{\text {eff }}$ (lept), Phys. Lett. B 394, 188 (1997).

[40] G. Degrassi, P. Gambino, M. Passera, and A. Sirlin, The role of $M_{W}$ in precision studies of the standard model, Phys. Lett. B 418, 209 (1998).

[41] M. Passera and A. Sirlin, Radiative corrections to $W$ and quark propagators in the resonance region, Phys. Rev. D 58, 113010 (1998).

[42] A. Freitas, W. Hollik, W. Walter, and G. Weiglein, Complete fermionic two loop results for the $M_{W}-M_{Z}$ interdependence, Phys. Lett. B 495, 338 (2000); Erratum, Phys. Lett. B 570, 265 (2003).

[43] A. Freitas, W. Hollik, W. Walter, and G. Weiglein, Electroweak two loop corrections to the $M_{W}-M_{Z}$ mass correlation in the standard model, Nucl. Phys. B632, 189 (2002); Erratum, Nucl. Phys. B666, 305 (2003).

[44] M. Awramik and M. Czakon, Complete Two Loop Bosonic Contributions to the Muon Lifetime in the Standard Model, Phys. Rev. Lett. 89, 241801 (2002).

[45] A. Onishchenko and O. Veretin, Two loop bosonic electroweak corrections to the muon lifetime and $M_{Z}-M_{W}$ interdependence, Phys. Lett. B 551, 111 (2003).
[46] M. Awramik, M. Czakon, A. Onishchenko, and O. Veretin, Bosonic corrections to $\Delta r$ at the two loop level, Phys. Rev. D 68, 053004 (2003).

[47] M. Faisst, J. H. Kühn, T. Seidensticker, and O. Veretin, Three loop top quark contributions to the $\rho$ parameter, Nucl. Phys. B665, 649 (2003).

[48] M. Awramik and M. Czakon, Complete two loop electroweak contributions to the muon lifetime in the standard model, Phys. Lett. B 568, 48 (2003).

[49] M. Awramik, M. Czakon, A. Freitas, and G. Weiglein, Precise prediction for the $W$ boson mass in the standard model, Phys. Rev. D 69, 053006 (2004).

[50] Y. Schroder and M. Steinhauser, Four-loop singlet contribution to the $\rho$ parameter, Phys. Lett. B 622, 124 (2005).

[51] K. G. Chetyrkin, M. Faisst, J. H. Kühn, P. Maierhofer, and C. Sturm, Four-Loop QCD Corrections to the $\rho$ Parameter, Phys. Rev. Lett. 97, 102003 (2006).

[52] R. Boughezal and M. Czakon, Single scale tadpoles and $\mathcal{O}\left(G_{F} m_{t}^{2} \alpha_{S}^{3}\right)$ corrections to the $\rho$ parameter, Nucl. Phys. B755, 221 (2006).

[53] F. Jegerlehner, M. Y. Kalmykov, and O. Veretin, $\overline{M S}$ versus pole masses of gauge bosons: Electroweak bosonic two loop corrections, Nucl. Phys. B641, 285 (2002).

[54] F. Jegerlehner, M. Y. Kalmykov, and O. Veretin, Full two loop electroweak corrections to the pole masses of gauge bosons, Nucl. Phys. B, Proc. Suppl. 116, 382 (2003).

[55] F. Jegerlehner, M. Y. Kalmykov, and O. Veretin, $\overline{M S}$ versus pole masses of gauge bosons: 2. Two loop electroweak fermion corrections, Nucl. Phys. B658, 49 (2003).

[56] G. Degrassi, P. Gambino, and P. P. Giardino, The $m_{W}-m_{Z}$ interdependence in the standard model: A new scrutiny, J. High Energy Phys. 05 (2015) 154.

[57] S. P. Martin, Pole mass of the $W$ boson at two-loop order in the pure $\overline{M S}$ scheme, Phys. Rev. D 91, 114003 (2015).

[58] S. P. Martin, Z-boson pole mass at two-loop order in the pure $\overline{M S}$ scheme, Phys. Rev. D 92, 014026 (2015).

[59] R. Tarrach, The pole mass in perturbative QCD, Nucl. Phys. B183, 384 (1981).

[60] N. Gray, D. J. Broadhurst, W. Grafe, and K. Schilcher, Three loop relation of quark $\overline{\mathrm{MS}}$ and pole masses, Z. Phys. C 48, 673 (1990).

[61] K. G. Chetyrkin and M. Steinhauser, Short Distance Mass of a Heavy Quark at Order $\alpha_{S}^{3}$, Phys. Rev. Lett. 83, 4001 (1999).

[62] K. G. Chetyrkin and M. Steinhauser, The relation between the $\overline{\mathrm{MS}}$ and the on-shell quark mass at order $\alpha_{S}^{3}$, Nucl. Phys. B573, 617 (2000).

[63] K. Melnikov and T. v. Ritbergen, The three loop relation between the $\overline{\mathrm{MS}}$ and the pole quark masses, Phys. Lett. B 482, 99 (2000).

[64] P. Marquard, A. V. Smirnov, V. A. Smirnov, and M. Steinhauser, Quark Mass Relations to Four-Loop Order in Perturbative QCD, Phys. Rev. Lett. 114, 142002 (2015).

[65] P. Marquard, A. V. Smirnov, V. A. Smirnov, M. Steinhauser, and D. Wellmann, $\overline{\mathrm{MS}}$-on-shell quark mass relation up to four loops in QCD and a general $\mathrm{SU}(N)$ gauge group, Phys. Rev. D 94, 074025 (2016).

[66] M. Beneke and V. M. Braun, Naive non-Abelianization and resummation of fermion bubble chains, Phys. Lett. B 348, 513 (1995). 
[67] P. Ball, M. Beneke, and V. M. Braun, Resummation of $\left(\beta_{0} \alpha_{s}\right)^{n}$ corrections in QCD: Techniques and applications to the $\tau$ hadronic width and the heavy quark pole mass, Nucl. Phys. B452, 563 (1995).

[68] A. L. Kataev and V.S. Molokoedov, On the flavour dependence of the $\mathcal{O}\left(\alpha_{s}^{4}\right)$ correction to the relation between running and pole heavy quark masses, Eur. Phys. J. Plus 131, 271 (2016); Multiloop contributions to the $\overline{\mathrm{MS}}$-onshell mass relation for heavy quarks in QCD and charged leptons in QED and the asymptotic structure of the perturbative QCD series, arXiv:1807.05406; Dependence of five- and six-loop estimated QCD corrections to the relation between pole and running masses of heavy quarks on the number of light flavours, arXiv:1811.02867.

[69] M. Beneke, P. Marquard, P. Nason, and M. Steinhauser, On the ultimate uncertainty of the top quark pole mass, Phys. Lett. B 775, 63 (2017).

[70] A. H. Hoang, C. Lepenik, and M. Preisser, On the light massive flavor dependence of the large order asymptotic behavior and the ambiguity of the pole mass, J. High Energy Phys. 09 (2017) 099.

[71] M. Bohm, H. Spiesberger, and W. Hollik, On the one loop renormalization of the electroweak standard model and its application to leptonic processes, Fortschr. Phys. 34, 687 (1986).

[72] R. Hempfling and B. A. Kniehl, On the relation between the fermion pole mass and MS Yukawa coupling in the standard model, Phys. Rev. D 51, 1386 (1995).

[73] F. Jegerlehner and M. Y. Kalmykov, $\mathcal{O}\left(\alpha \alpha_{S}\right)$ correction to the pole mass of the $t$-quark within the standard model, Nucl. Phys. B676, 365 (2004).

[74] F. Jegerlehner and M. Y. Kalmykov, $\mathcal{O}\left(\alpha \alpha_{S}\right)$ relation between pole- and $\overline{\mathrm{MS}}$ mass of the $t$-quark, Acta Phys. Pol. B 34, 5335 (2003).

[75] M. Faisst, J. H. Kühn, and O. Veretin, Pole versus $\overline{\mathrm{MS}}$ mass definitions in the electroweak theory, Phys. Lett. B 589, 35 (2004).

[76] D. Eiras and M. Steinhauser, Two-loop $\mathcal{O}\left(\alpha \alpha_{S}\right)$ corrections to the on-shell fermion propagator in the standard model, J. High Energy Phys. 02 (2006) 010.

[77] F. Jegerlehner, M. Y. Kalmykov, and B. A. Kniehl, On the difference between the pole and the $\overline{\mathrm{MS}}$ masses of the top quark at the electroweak scale, Phys. Lett. B 722, 123 (2013).

[78] S. P. Martin, Fermion self-energies and pole masses at twoloop order in a general renormalizable theory with massless gauge bosons, Phys. Rev. D 72, 096008 (2005).

[79] B. A. Kniehl and O.L. Veretin, Two-loop electroweak threshold corrections to the bottom and top Yukawa couplings, Nucl. Phys. B885, 459 (2014); Erratum, Nucl. Phys. B894, 56 (2015).

[80] S. P. Martin, Top-quark pole mass in the tadpole-free $\overline{M S}$ scheme, Phys. Rev. D 93, 094017 (2016).

[81] M. E. Machacek and M. T. Vaughn, Two loop renormalization group equations in a general quantum field theory: 1. Wave function renormalization, Nucl. Phys. B222, 83 (1983).

[82] M. E. Machacek and M. T. Vaughn, Two loop renormalization group equations in a general quantum field theory: 2. Yukawa couplings, Nucl. Phys. B236, 221 (1984).
[83] I. Jack and H. Osborn, General background field calculations with fermion fields, Nucl. Phys. B249, 472 (1985).

[84] M. E. Machacek and M. T. Vaughn, Two loop renormalization group equations in a general quantum field theory: 3. Scalar quartic couplings, Nucl. Phys. B249, 70 (1985).

[85] M. x. Luo and Y. Xiao, Two Loop Renormalization Group Equations in the Standard Model, Phys. Rev. Lett. 90, 011601 (2003).

[86] L. N. Mihaila, J. Salomon, and M. Steinhauser, Gauge Coupling Beta Functions in the Standard Model to Three Loops, Phys. Rev. Lett. 108, 151602 (2012).

[87] K. G. Chetyrkin and M. F. Zoller, Three-loop $\beta$-functions for top-Yukawa and the Higgs self-interaction in the standard model, J. High Energy Phys. 06 (2012) 033.

[88] A. V. Bednyakov, A. F. Pikelner, and V. N. Velizhanin, Anomalous dimensions of gauge fields and gauge coupling beta-functions in the standard model at three loops, J. High Energy Phys. 01 (2013) 017.

[89] A. V. Bednyakov, A. F. Pikelner, and V. N. Velizhanin, Yukawa coupling beta-functions in the standard model at three loops, Phys. Lett. B 722, 336 (2013).

[90] K. G. Chetyrkin and M. F. Zoller, $\beta$-function for the Higgs self-interaction in the standard model at three-loop level, J. High Energy Phys. 04 (2013) 091.

[91] A. V. Bednyakov, A. F. Pikelner, and V. N. Velizhanin, Higgs self-coupling beta-function in the standard model at three loops, Nucl. Phys. B875, 552 (2013).

[92] A. V. Bednyakov, A. F. Pikelner, and V. N. Velizhanin, Three-loop Higgs self-coupling beta-function in the standard model with complex Yukawa matrices, Nucl. Phys. B879, 256 (2014).

[93] A. V. Bednyakov, A. F. Pikelner, and V. N. Velizhanin, Three-loop SM beta-functions for matrix Yukawa couplings, Phys. Lett. B 737, 129 (2014).

[94] K. G. Chetyrkin and M. F. Zoller, Leading QCD-induced four-loop contributions to the $\beta$-function of the Higgs selfcoupling in the SM and vacuum stability, J. High Energy Phys. 06 (2016) 175.

[95] T. van Ritbergen, J. A. M. Vermaseren, and S. A. Larin, The four loop beta function in quantum chromodynamics, Phys. Lett. B 400, 379 (1997).

[96] M. Czakon, The four-loop QCD beta-function and anomalous dimensions, Nucl. Phys. B710, 485 (2005).

[97] P. A. Baikov, K. G. Chetyrkin, and J. H. Kühn, Five-Loop Running of the QCD Coupling Constant, Phys. Rev. Lett. 118, 082002 (2017).

[98] F. Herzog, B. Ruijl, T. Ueda, J. A. M. Vermaseren, and A. Vogt, The five-loop beta function of Yang-Mills theory with fermions, J. High Energy Phys. 02 (2017) 090.

[99] O. V. Tarasov, Anomalous dimensions of quark masses in three loop approximation, Report No. JINR-P2-82-900, 1982 (unpublished).

[100] K. G. Chetyrkin, Quark mass anomalous dimension to $\mathcal{O}\left(\alpha_{S}^{4}\right)$, Phys. Lett. B 404, 161 (1997).

[101] J. A. M. Vermaseren, S. A. Larin, and T. van Ritbergen, The four loop quark mass anomalous dimension and the invariant quark mass, Phys. Lett. B 405, 327 (1997).

[102] P. A. Baikov, K. G. Chetyrkin, and J. H. Kühn, Quark mass and field anomalous dimensions to $\mathcal{O}\left(\alpha_{S}^{5}\right)$, J. High Energy Phys. 10 (2014) 076. 
[103] S. Weinberg, Effective gauge theories, Phys. Lett. 91B, 51 (1980); B. A. Ovrut and H. J. Schnitzer, The decoupling theorem and minimal subtraction, Phys. Lett. 100B, 403 (1981).

[104] W. Bernreuther and W. Wetzel, Decoupling of heavy quarks in the minimal subtraction scheme, Nucl. Phys. B197, 228 (1982); Erratum, Nucl. Phys. B513, 758 (1998).

[105] S. A. Larin, T. van Ritbergen, and J. A. M. Vermaseren, The large quark mass expansion of $\Gamma\left(Z^{0} \rightarrow\right.$ hadrons $)$ and $\Gamma\left(\tau^{-} \rightarrow\right.$ $\nu_{\tau}+$ hadrons) in the order $\alpha_{S}^{3}$, Nucl. Phys. B438, 278 (1995).

[106] K. G. Chetyrkin, B. A. Kniehl, and M. Steinhauser, Decoupling relations to $\mathcal{O}\left(\alpha_{S}^{3}\right)$ and their connection to lowenergy theorems, Nucl. Phys. B510, 61 (1998).

[107] A. G. Grozin, M. Hoeschele, J. Hoff, and M. Steinhauser, Simultaneous decoupling of bottom and charm quarks, J. High Energy Phys. 09 (2011) 066.

[108] Y. Schroder and M. Steinhauser, Four-loop decoupling relations for the strong coupling, J. High Energy Phys. 01 (2006) 051.

[109] K. G. Chetyrkin, J. H. Kühn, and C. Sturm, QCD decoupling at four loops, Nucl. Phys. B744, 121 (2006).

[110] A. V. Bednyakov, On the electroweak contribution to the matching of the strong coupling constant in the SM, Phys. Lett. B 741, 262 (2015).

[111] T. Liu and M. Steinhauser, Decoupling of heavy quarks at four loops and effective Higgs-fermion coupling, Phys. Lett. B 746, 330 (2015).

[112] K. G. Chetyrkin, J. H. Kühn, and M. Steinhauser, RunDec: A Mathematica package for running and decoupling of the strong coupling and quark masses, Comput. Phys. Commun. 133, 43 (2000); B. Schmidt and M. Steinhauser, CRunDec: A C++ package for running and decoupling of the strong coupling and quark masses, Comput. Phys. Commun. 183, 1845 (2012); F. Herren and M. Steinhauser, Version 3 of RunDec and CRunDec, Comput. Phys. Commun. 224, 333 (2018).

[113] W. J. Marciano and J. L. Rosner, Atomic Parity Violation as a Probe of New Physics, Phys. Rev. Lett. 65, 2963 (1990); Erratum, Phys. Rev. Lett. 68, 898 (1992).

[114] G. Degrassi, S. Fanchiotti, and A. Sirlin, Relations between the on-shell and $\overline{\mathrm{MS}}$ frameworks and the $M_{W}-M_{Z}$ interdependence, Nucl. Phys. B351, 49 (1991).

[115] S. Fanchiotti, B. A. Kniehl, and A. Sirlin, Incorporation of QCD effects in basic corrections of the electroweak theory, Phys. Rev. D 48, 307 (1993).

[116] S. Eidelman and F. Jegerlehner, Hadronic contributions to $g-2$ of the leptons and to the effective fine structure constant $\alpha\left(M_{Z}^{2}\right)$, Z. Phys. C 67, 585 (1995).

[117] H. Burkhardt and B. Pietrzyk, Update of the hadronic contribution to the QED vacuum polarization, Phys. Lett. B 356, 398 (1995).

[118] A. D. Martin and D. Zeppenfeld, A determination of the QED coupling at the Z pole, Phys. Lett. B 345, 558 (1995).

[119] R. Alemany, M. Davier, and A. Hocker, Improved determination of the hadronic contribution to the muon $g-2$ and to $\alpha\left(M_{Z}\right)$ using new data from hadronic tau decays, Eur. Phys. J. C 2, 123 (1998).

[120] M. Davier and A. Hocker, Improved determination of $\alpha\left(M_{Z}^{2}\right)$ and the anomalous magnetic moment of the muon, Phys. Lett. B 419, 419 (1998).
[121] J. H. Kühn and M. Steinhauser, A theory driven analysis of the effective QED coupling at $M_{Z}$, Phys. Lett. B 437, 425 (1998).

[122] M. Steinhauser, Leptonic contribution to the effective electromagnetic coupling constant up to three loops, Phys. Lett. B 429, 158 (1998).

[123] J. Erler, Calculation of the QED coupling $\alpha\left(M_{Z}\right)$ in the modified minimal subtraction scheme, Phys. Rev. D 59, 054008 (1999).

[124] J. Erler, Global fits to electroweak data using GAPP, arXiv: hep-ph/0005084.

[125] G. Degrassi and A. Vicini, Two loop renormalization of the electric charge in the standard model, Phys. Rev. D 69, 073007 (2004).

[126] M. Tanabashi et al. (Particle Data Group), Review of particle physics, Phys. Rev. D 98, 030001 (2018).

[127] M. Davier, A. Hoecker, B. Malaescu, and Z. Zhang, Reevaluation of the hadronic vacuum polarisation contributions to the standard model predictions of the muon $g-2$ and $\alpha\left(m_{Z}^{2}\right)$ using newest hadronic cross-section data, Eur. Phys. J. C 77, 827 (2017).

[128] F. Jegerlehner, Variations on photon vacuum polarization, arXiv:1711.06089.

[129] A. Keshavarzi, D. Nomura, and T. Teubner, Muon $g-2$ and $\alpha\left(M_{Z}^{2}\right)$ : A new data-based analysis, Phys. Rev. D 97, 114025 (2018).

[130] B. A. Kniehl, J. H. Piclum, and M. Steinhauser, Relation between bottom-quark $\overline{\mathrm{MS}}$ Yukawa coupling and pole mass, Nucl. Phys. B695, 199 (2004).

[131] A. V. Bednyakov, B. A. Kniehl, A. F. Pikelner, and O. L. Veretin, On the $b$-quark running mass in QCD and the SM, Nucl. Phys. B916, 463 (2017).

[132] A. I. Davydychev and J. B. Tausk, Two loop self-energy diagrams with different masses and the momentum expansion, Nucl. Phys. B397, 123 (1993); A. I. Davydychev, V. A. Smirnov, and J. B. Tausk, Large momentum expansion of two loop self-energy diagrams with arbitrary masses, Nucl. Phys. B410, 325 (1993); F. A. Berends and J. B. Tausk, On the numerical evaluation of scalar two loop self-energy diagrams, Nucl. Phys. B421, 456 (1994).

[133] B. A. Kniehl, A. V. Kotikov, A. I. Onishchenko, and O. L. Veretin, Strong-Coupling Constant with Flavor Thresholds at Five Loops in the $\overline{\mathrm{MS}}$ Scheme, Phys. Rev. Lett. 97, 042001 (2006).

[134] S. Bekavac, A. Grozin, D. Seidel, and M. Steinhauser, Light quark mass effects in the on-shell renormalization constants, J. High Energy Phys. 10 (2007) 006.

[135] S. Bekavac, A. G. Grozin, D. Seidel, and V. A. Smirnov, Three-loop on-shell Feynman integrals with two masses, Nucl. Phys. B819, 183 (2009).

[136] S. P. Martin, Refined gluino and squark pole masses beyond leading order, Phys. Rev. D 74, 075009 (2006).

[137] P. A. Baikov, K. G. Chetyrkin, J. H. Kühn, and J. Rittinger, Vector correlator in massless QCD at order $\mathcal{O}\left(\alpha_{S}^{4}\right)$ and the QED beta-function at five loop, J. High Energy Phys. 07 (2012) 017.

[138] See Supplemental Material at http://link.aps.org/ supplemental/10.1103/PhysRevD.99.033007, for [brief description]. 